\title{
Genomic Characterization and Expression of Juvenile Hormone Esterase-Like Carboxylesterase Genes in Pacific White Shrimp, Litopenaeus vannamei
}

\author{
Xiaoxi Zhang ${ }^{1,2,3,4}$, Jianbo Yuan ${ }^{1,2,3, *}$, Xiaojun Zhang ${ }^{1,2,3, *}$, Jianhai Xiang ${ }^{1,2,3}$ and Fuhua Li ${ }^{1,2,3}$ \\ 1 CAS Key Laboratory of Experimental Marine Biology, Institute of Oceanology, Chinese Academy of Sciences, \\ Qingdao 266071, China; zhangxiaoxi@qdio.ac.cn (X.Z.); jhxiang@qdio.ac.cn (J.X.); fhli@qdio.ac.cn (F.L.) \\ 2 Laboratory for Marine Biology and Biotechnology, Qingdao National Laboratory for \\ Marine Science and Technology, Qingdao 266237, China \\ 3 Center for Ocean Mega-Science, Chinese Academy of Sciences, Qingdao 266071, China \\ 4 University of Chinese Academy of Sciences, Beijing 100049, China \\ * Correspondence: yuanjb@qdio.ac.cn (J.Y.); xjzhang@qdio.ac.cn (X.Z.)
}

Received: 18 June 2020; Accepted: 29 July 2020; Published: 30 July 2020

\begin{abstract}
The sesquiterpenoid methyl farnesoate (MF), a juvenile hormone $(\mathrm{JH})$ analog, plays important roles in many physiological processes of crustaceans, such as morphogenesis, molting and reproduction. Juvenile hormone esterase-like (JHE-like) carboxylesterase (CXE) is a key enzyme in MF degradation, playing a significant role in regulating MF titer. However, its function is barely known in shrimp. In this study, a total of 21 JHE-like CXEs (LvCXEs) were characterized in Pacific white shrimp Litopenaeus vannamei, based on the full genome and multi-transcriptomic data. LvCXE has a conserved triplet catalytic site (Ser-Glu-His) and a characteristic GxSxG motif. Most LvCXEs were highly expressed in the hepatopancreas, which was the main site for MF degradation. LvCXEs containing a GESAG motif showed a specific expansion in the L. vannamei genome. Those GESAG-containing LvCXEs presented differential expressions at different larvae stages and different molting stages of L. vannamei, which suggested their potential functions in development and molting. Additionally, when the transcription level of CXEs was inhibited, it could lead to failed molt and death of L. vannamei. When we further detected the expression levels of the key ecdysone responsive transcription factors including $L v E 75, L v B r-C, L v H r 3$ and $L v F t z-f 1$ after the CXE inhibitor was injected into L. vannamei, they all showed apparent down-regulation. These results suggested that the expansion of LvCXEs in the L. vannamei genome should contribute to the regulation of metamorphosis at larvae stages and frequent molting during the growth of L. vannamei.
\end{abstract}

Keywords: juvenile hormone esterase-like carboxylesterase; development; molting; penaeid shrimp

\section{Introduction}

Carboxylesterase is a superfamily of multifunctional enzyme which ubiquitously exists in animals, plants and microbes [1,2]. Based on sequence similarity and substrate specificity, carboxylesterases can be divided into eight subfamilies- $\alpha$-esterases (Ae), $\beta$-esterases (Be), juvenile hormone esterases (JHE), gliotactins (Gli), acetylcholinesterases (AChE), neurotactins (Nrt), neuroligins (Nrl) and glutactins (Glt) [3]. Among them, JHE is considered to be the predominant enzyme involved in degradation of juvenile hormone $(\mathrm{JH})$, which is a group of sesquiterpenoid compounds with pleiotropic functions in development, metamorphosis, molting, growth, reproduction and pheromone biosynthesis of insects $[4,5]$. JHE converts $\mathrm{JH}$ to $\mathrm{JH}$ acid and then regulates $\mathrm{JH}$ titer at appropriate levels, and thereby directs development and metamorphosis in insects [6,7]. The increase of JHE activity at late developmental stage is one of the important biochemical events that leads to pupation $[8,9]$. 
Moreover, inhibition of JHE activity maintained JH titer, led to abnormally large larvae and delayed metamorphosis [10,11].

$\mathrm{JH}$ has never been identified in crustaceans, and the only JH-like compound, named as methyl farnesoate (MF), is an unepoxidated form of insect JH Ш[12]. MF is produced in the mandibular organs (MOs) of crustaceans and possesses similar functions with insect $\mathrm{JH}$, which is involved in many crucial physiological processes such as morphogenesis, molting, growth and reproduction etc. [12-15]. For example, exposure of lobster larvae to MF increased the duration of their development [16], and addition of MF to the feed of freshwater prawn retarded the growth and development at late larval stages [17]. Meanwhile, the role of MF during the molting cycle was uncertain. For example, MF can accelerate molting in crayfish Cherax quandricarinatus [18] and Procambarus clarkii [15], but causes molt-related mortality and delay the molting process of Artemia larvae [19]. The regulation of the molting process is complicated, and it experiences the following processes. The titer of MF and ecdysone (molting hormone, a steroid compound derived from cholesterol) presents periodic fluctuation in hemolymph of adult crustaceans [20-22]. In detail, MF and ecdysone titer increases gradually at the early pre-molt stage and reaches a maximum near molting, then decreases rapidly at the late pre-molt stage. It leads to large scale synthesis of ecdysone in $\mathrm{Y}$ organ at the pre-molt stage owing to the negative feedback [23]. Meanwhile, the expressions of ecdysone receptor (EcR) and retinoid $X$ receptor (RXR), which form a heterodimer to which ecdysone binds, are induced [24]. As early response genes, Broad-complex (Br-C), E74 and E75 are then normally up-regulated by EcR. After that, several early late genes such as hormone receptor $3(\mathrm{Hr} 3)$ and late response genes such as Ftz-f1, are expressed [25]. Finally, the molting process occurs. Therefore, a rapid decrease of both MF and ecdysone titer in hemolymph near molting is the key factor for successful molting [5]. When injecting 20E into crayfish at the late pre-molt stage, the synthesis of ecdysone in $\mathrm{Y}$ organ decreased and the normal molting cycle of crayfish delayed [26].

There are many ways to study the function of JHE in insects. For example, gene knockout, RNA interference and inhibitor injection. Among them, OTFP (3-octylthio-1,1,1-trifluoropropan2-propanone), a slow and tight-binding inhibitor of JHE, was widely used to investigate the function of JHE owing to the fact that it can suppress more than $98 \%$ of the JHE activity in insects $[27,28]$. Furthermore, OTFP had also been used in crustaceans, such as the spider crab Libinia emarginata [29]. However, due to the weakness of the experimental platform, the study on the function of juvenile hormone esterase-like (JHE-like) carboxylesterases (CXE) in crustaceans is limited. Like insects, CXE was supposed to degrade MF through ester hydrolysis in crustaceans, and it was supported by the results that the expression of CXE genes was up-regulated by eyestalk ablation or MF treatment in several crustacean species [30-33]. Nevertheless, they mainly focused on the role of CXE in reproductive development and the innate immune response [30-33], whereas their functions in early development and the molting process are barely known in crustaceans. The Pacific white shrimp Litopenaeus vannamei is one of the most economically important marine aquaculture species in the world (FAO). Additionally, the penaeid shrimp experiences about 50 molts during a lifetime [34], far more than other arthropods [35]. The expansion of many molt-related gene families, such as Broad-Complex (Br-C), the crustacean hyperglycemic hormone $(\mathrm{CHH})$ and chitinase family, contributed to frequent molting of L. vannamei. However, a previous study has also found that the CXE gene family showed expansion in L. vannamei genome through comparative genomic analysis [36], whereas the relationship between the CXE gene family and molting process of L. vannamei remains uncertain. In this study, we characterized LvCXEs in L. vannamei genome comprehensively and conducted phylogenetic analysis on these LvCXEs. We then illustrated their expressions at early developmental stages and investigated the relationships between the expansion of the CXE gene family and frequent molting of L. vannamei. The OTFP inhibition experiment was performed to validate their functions in the molting process of L. vannamei. The results obtained in this study will enable us to better understand the function of CXEs during the early developmental stages and molt cycle of L. vannamei and even other crustaceans. 


\section{Results}

\subsection{Characterization of CXE Genes in L.vannamei genome}

Among 51 CXE genes, a total of 21 LvCXEs held a complete open reading frame (ORF), which were named as LvCXE1-LvCXE21, respectively. The predicted proteins of these LvCXEs varied from 488 to 669 amino acid residues. The estimated Mw ranged from 54.3 to $74.5 \mathrm{kDa}$, with pI of 4.53 to 8.98 (Table 1). The exon numbers of all LvCXEs varied from 8 to 13 (Figure 1). LvCXE2 and LvCXE3 were distributed in scaffold883, LvCXE4 and LvCXE5 located in scaffold962, LvCXE9 and LvCXE10 were distributed in scaffold1839. Other LvCXEs scattered in L. vannamei genome (Table 1).

Table 1. Characteristics of $21 \mathrm{LvCXEs}$ identified in penaeid shrimp genome.

\begin{tabular}{|c|c|c|c|c|}
\hline Gene ID & Amino Acids & Mw (kD) & pI & Location \\
\hline LvCXE1 & 564 & 61.4 & 5.07 & LVANscaffold_866: 18624-23041 \\
\hline LvCXE2 & 488 & 54.3 & 6.55 & LVANscaffold_883: 576355-581238 \\
\hline LvCXE3 & 574 & 64.0 & 5.69 & LVANscaffold_883: 575275-603536 \\
\hline LvCXE4 & 577 & 64.7 & 5.58 & LVANscaffold_962: 831167-839422 \\
\hline LvCXE5 & 575 & 63.5 & 6.03 & LVANscaffold_962: 839832-855606 \\
\hline LvCXE6 & 544 & 60.0 & 4.78 & LVANscaffold_1108: 73812-101034 \\
\hline LvCXE7 & 569 & 63.2 & 4.88 & LVANscaffold_1123: 580276-596930 \\
\hline$L v C X E 8$ & 585 & 64.3 & 5.79 & LVANscaffold_1168: 597704-610937 \\
\hline LvCXE9 & 581 & 65.1 & 5.34 & LVANscaffold_1839: 157651-172084 \\
\hline LvCXE10 & 573 & 63.3 & 5.13 & LVANscaffold_1839: 184142-188709 \\
\hline LvCXE11 & 585 & 64.8 & 5.60 & LVANscaffold_1907: 1025753-1038397 \\
\hline LvCXE12 & $>605$ & 66.6 & 5.75 & LVANscaffold_2316: 553488-572600 \\
\hline LvCXE13 & 669 & 74.5 & 4.95 & LVANscaffold_2381: 397192-408701 \\
\hline LvCXE14 & 572 & 64.1 & 8.98 & LVANscaffold_2639: 313127-321369 \\
\hline LvCXE15 & 561 & 62.1 & 5.77 & LVANscaffold_2654: 959807-971335 \\
\hline LvCXE16 & 565 & 62.2 & 4.97 & LVANscaffold_2937: 352862-369967 \\
\hline LvCXE17 & 621 & 69.8 & 4.53 & LVANscaffold_2938: 299225-309403 \\
\hline LvCXE18 & 627 & 68.1 & 6.45 & LVANscaffold_3071: 91750-98940 \\
\hline LvCXE19 & 561 & 62.1 & 6.40 & LVANscaffold_3486: 45723-66803 \\
\hline LvCXE20 & 592 & 65.7 & 6.10 & LVANscaffold_199: 131030-134929 \\
\hline LvCXE21 & 583 & 65.3 & 6.08 & LVANscaffold_2382: 54122-66300 \\
\hline
\end{tabular}
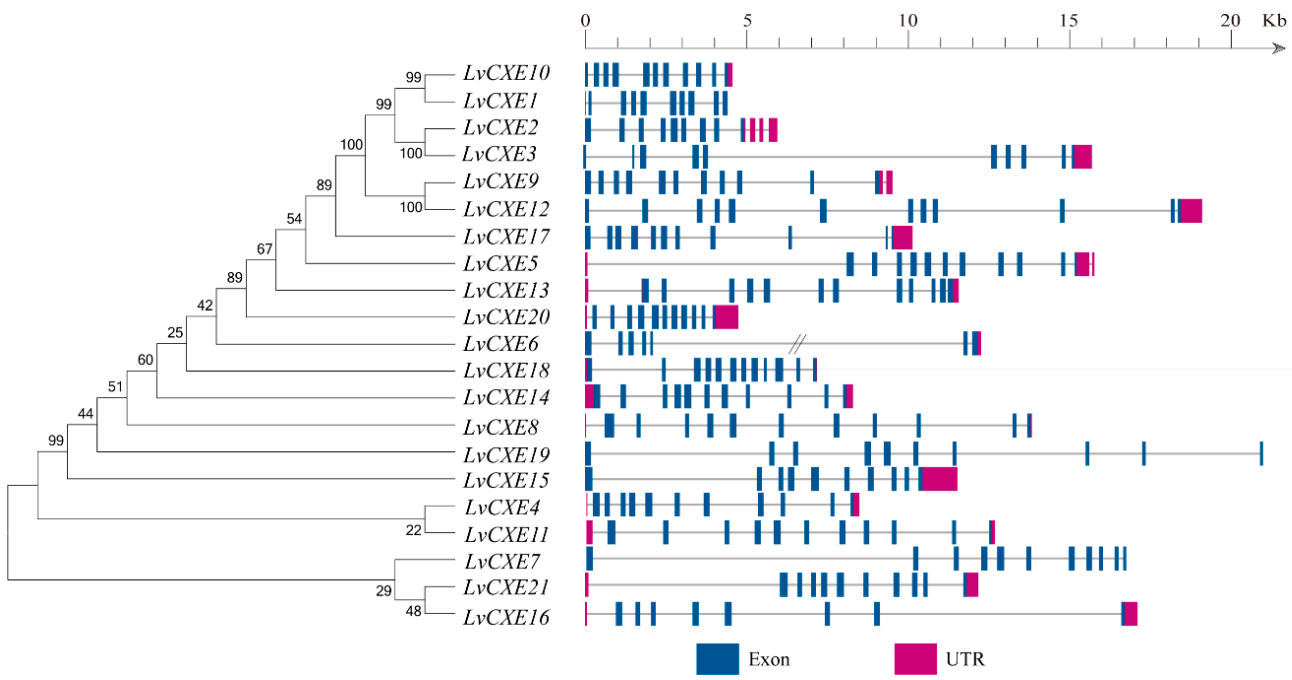

Figure 1. Schematic representation of exon-intron structures of the identified LvCXEs. The maximum likelihood (ML) phylogenetic tree on the left was generated using RAxML software with 100 bootstrap replicates. Exons, introns and untranslated regions (UTRs) are shown as blue rectangles, grey lines and rose red rectangles, respectively. Double slash represents a gap of genome scaffold. 


\subsection{Structure Analysis of LvCXEs}

A total of 15 of 21 LvCXEs contained ten motifs (Figure 2), indicating that their protein sequences showed high similarity. Among ten motifs, motif 5, 4, 1, 3, 6 contained the RF, GG, DQ, GxSxG, E/D motif, respectively, which were mentioned in Table 2. Except for the incomplete LvCXE12, all LvCXEs were expected to contain a signal peptide sequence (17-26 aa) at the N-terminal (Table 2). The multiple alignment revealed that all LvCXEs had a typical domain organization, including a conserved catalytic triad (Ser-Glu-His) and a carboxylesterase-specific GxSxG motif. A total of 14 LvCXEs contained the GESAG motif, and the other LvCXEs were different. In addition, LvCXEs had RF (arginine, phenylalanine) and DQ (aspartic acid, glutamine) residues at the same position as insect JHEs (Figure 3 and Table 2).

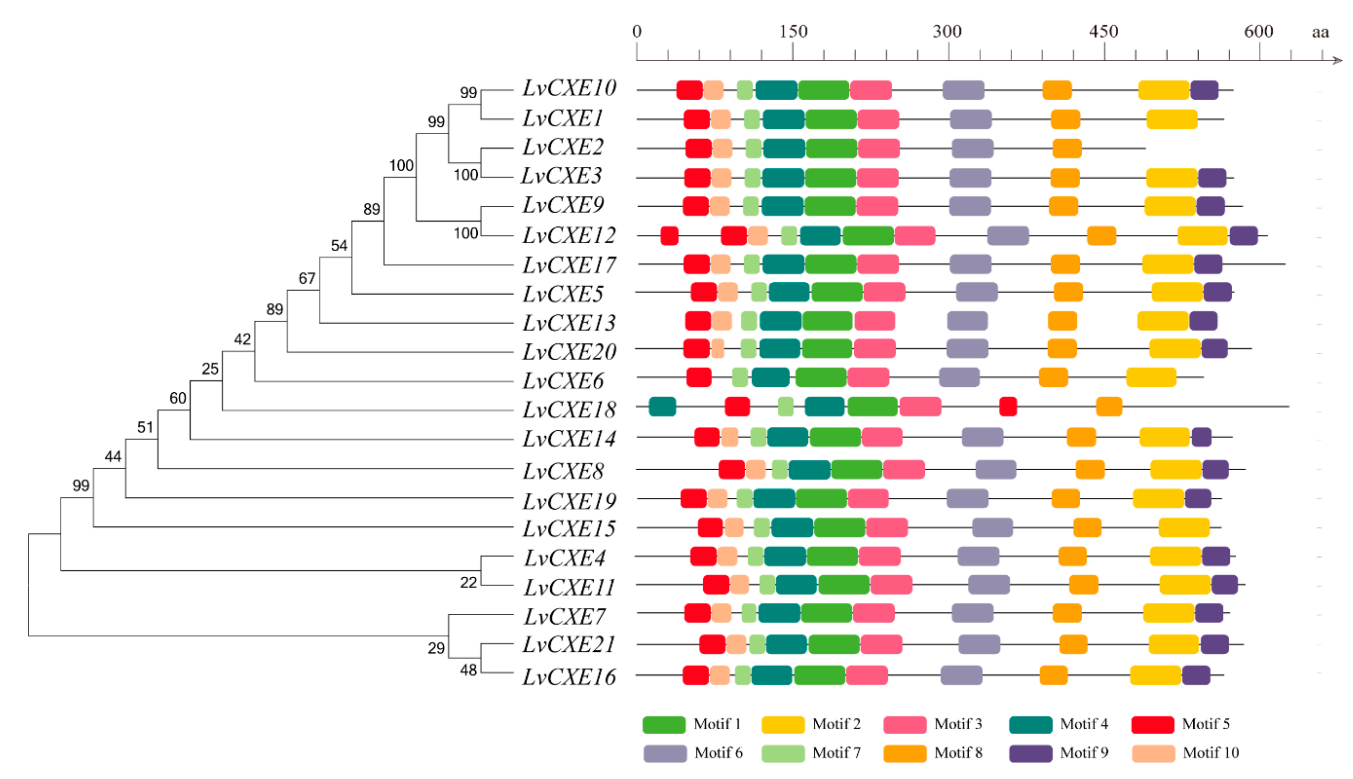

Figure 2. Schematic representation of conserved motifs identified in LvCXEs. The ML phylogenetic tree on the left was generated using RAxML software with 100 bootstrap replicates. Different color boxes represent different types of motifs, and the names of all members are shown on the bottom.

Table 2. The conserved motif organization of LvCXEs.

\begin{tabular}{|c|c|c|c|c|c|c|}
\hline Gene ID & Signal Peptide & RF & DQ & GxSxG & E/D & $\mathbf{H}$ \\
\hline LvCXE1 & + & RL & DQ & GESAG & $\mathrm{D}$ & $\mathrm{H}$ \\
\hline LvCXE2 & + & RL & DQ & GESAG & $\mathrm{E}$ & l \\
\hline LvCXE3 & + & RL & DQ & GESAG & $\mathrm{D}$ & $\mathrm{H}$ \\
\hline LvCXE4 & + & RF & DQ & GESAG & $\mathrm{E}$ & $\mathrm{H}$ \\
\hline LvCXE5 & + & RL & DQ & GQSAG & $\mathrm{E}$ & $\mathrm{H}$ \\
\hline LvCXE6 & + & RF & DQ & GGSAG & $\mathrm{E}$ & $\mathrm{H}$ \\
\hline LvCXE7 & + & RF & DQ & GESAG & $\mathrm{D}$ & $\mathrm{H}$ \\
\hline LvCXE8 & + & RL & DQ & GESAG & $\mathrm{E}$ & $\mathrm{H}$ \\
\hline LvCXE9 & + & KL & DQ & GESAG & $\mathrm{E}$ & $\mathrm{H}$ \\
\hline LvCXE10 & + & RL & DQ & GESAG & $\mathrm{D}$ & $\mathrm{H}$ \\
\hline LvCXE11 & + & RF & DQ & GESAG & $\mathrm{E}$ & $\mathrm{H}$ \\
\hline LvCXE12 & - & RF & DQ & GESAG & E & $\mathrm{H}$ \\
\hline LvCXE13 & + & RF & $\mathrm{DQ}$ & GESAG & E & $\mathrm{H}$ \\
\hline LvCXE14 & + & RF & $\mathrm{DQ}$ & GLSAG & E & $\mathrm{H}$ \\
\hline LvCXE15 & + & RF & DQ & GVSAG & E & $\mathrm{H}$ \\
\hline LvCXE16 & + & RF & DQ & GESAG & E & $\mathrm{H}$ \\
\hline LvCXE17 & + & RL & DQ & GESAG & E & $\mathrm{H}$ \\
\hline LvCXE18 & + & RW & DQ & GFSAG & E & $\mathrm{H}$ \\
\hline LvCXE19 & + & RF & DQ & GVSSG & E & $\mathrm{H}$ \\
\hline LvCXE20 & + & RF & DQ & GISAG & $\mathrm{D}$ & $\mathrm{H}$ \\
\hline LvCXE21 & + & RF & DQ & GESAG & E & $\mathrm{H}$ \\
\hline
\end{tabular}




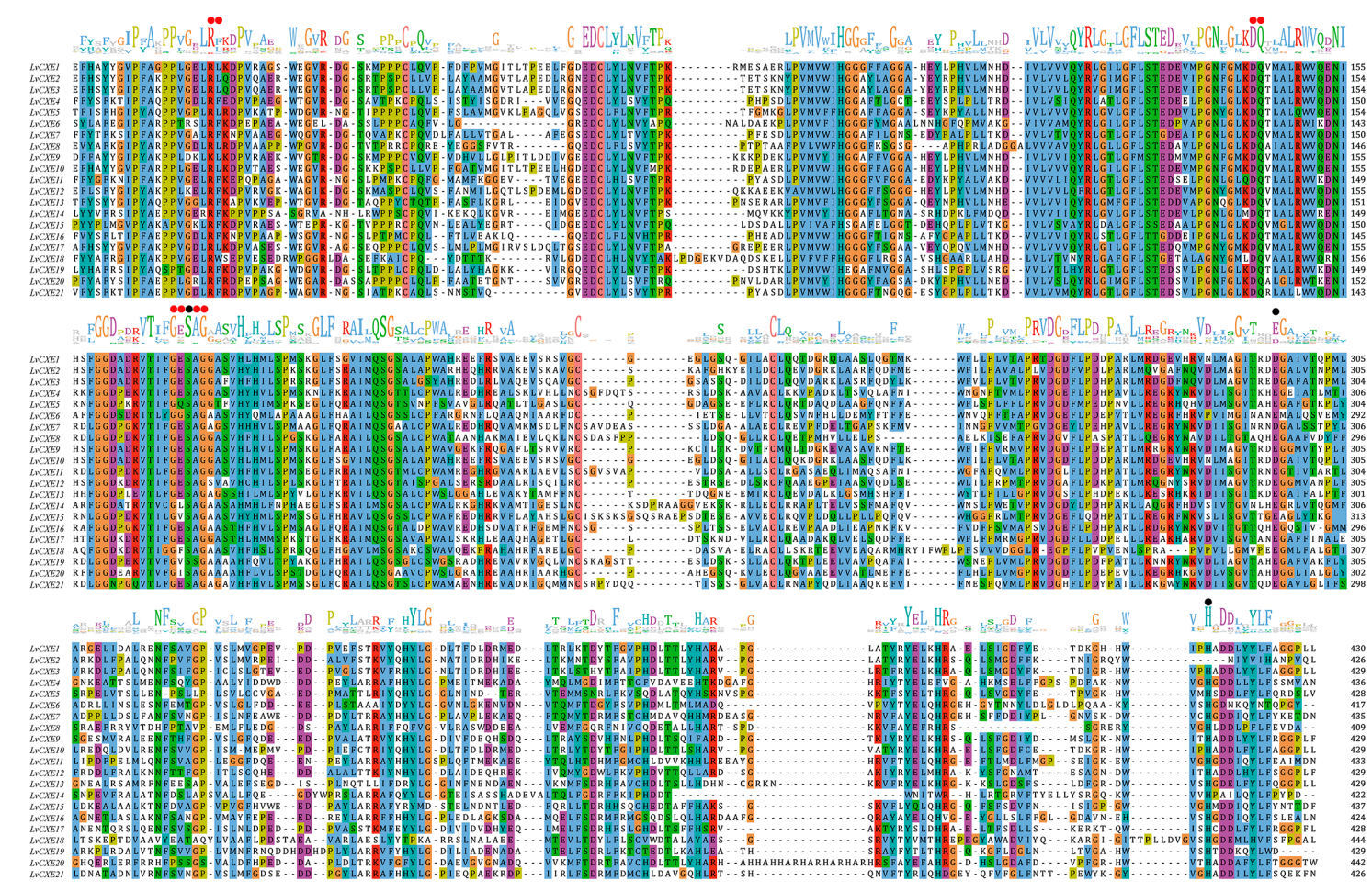

Figure 3. Multiple alignment of amino acid sequences containing conserved motifs and domains of LvCXEs. Black circles indicate the catalytic triad residues $(\mathrm{S}, \mathrm{E}, \mathrm{H})$ and red circles indicate conserved motifs of carboxylesterases.

\subsection{Phylogenetic Analysis}

The phylogenetic analysis was conducted to investigate the evolutionary relationship between LvCXEs, insect JHEs and other crustacean CXEs. The results showed that all LvCXEs were clustered within the monophyletic clade of crustacean CXE (Figure 4). Meanwhile, JHE genes of insect were clustered in another monophyletic clade. Additionally, most LvCXEs containing the GESAG motif were closely related to each other in the phylogenetic tree, suggesting that GESAG-containing CXE genes expanded specifically in L. vannamei. Furthermore, most of JHE genes of insect contained the GQSAG motif rather than GESAG.

\subsection{Spatial and Temporal Expression of LvCXEs}

RNA-seq data was used to analyze the spatial expression patterns of LvCXEs in 16 different tissues of the adult $L$. vannamei. The hierarchical clustering analysis showed that the expression patterns of LvCXEs can be divided into 4 modules (Figure 5). Module 1, including LvCXE2, LvCXE9, LvCXE10, $L v C X E 15$ and LvCXE21, was found to be expressed in almost all tissues detected, with exception of ovary, testis and muscle. Module 2, including LvCXE12, LvCXE17, LvCXE19, was also expressed in all tissues detected, but the expressions was lower than group 1. Module 3, including LvCXE3, LvCXE4, LvCXE5, LvCXE8, LvCXE14, LvCXE16 and LvCXE18, showed expression in specific tissues. For example, LvCXE4, LvCXE5 and LvCXE18 were almost specifically expressed in stomach, gill and antennary gland, respectively. Module 4, including LvCXE1, LvCXE6, LvCXE7, LvCXE11, LvCXE13 and LvCXE20, was highly expressed in hepatopancreas.

We also assessed the expression profiles of LvCXEs at early developmental stages of L. vannamei. Obviously, the expression levels of all LvCXEs could be clustered into two modules. Module 1 included LvCXE4, LvCXE6, LvCXE9, LvCXE10, LvCXE11, LvCXE13, LvCXE15, LvCXE16, LvCXE19 and LvCXE21, in which contained seven GESAG-containing genes. They showed high expressions from zoea I (Z1) to post-larva (P1) stage. In detail, their expressions increased until mysis II (M2) stage and decreased in 
mysis III (M3) stage, which was the end of metamorphosis. Other LvCXEs were clustered in module 2, whose expression levels were relatively low during the whole early developmental stages (Figure 6).

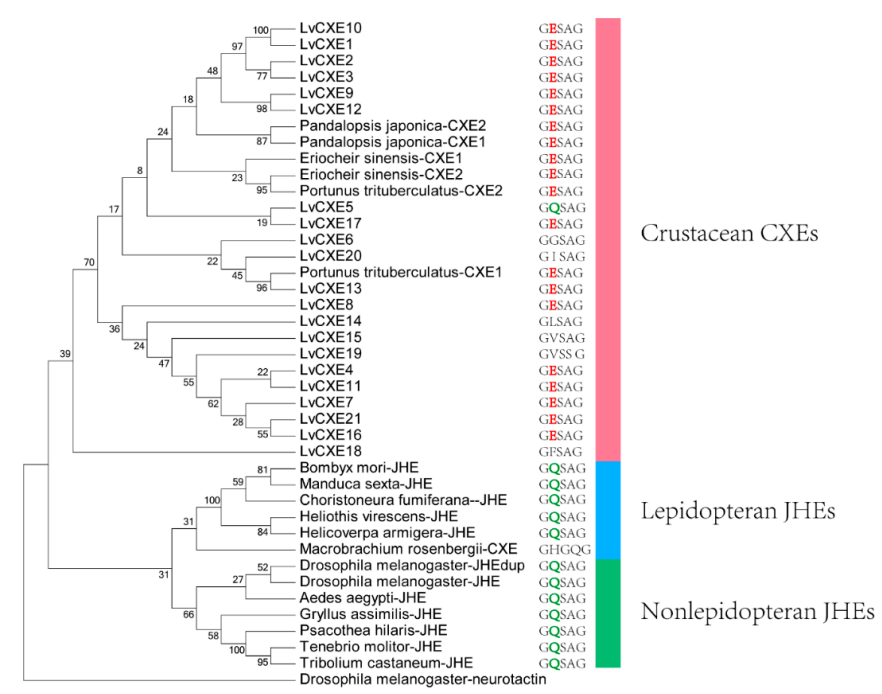

Figure 4. The ML phylogenetic tree of juvenile hormone esterases (JHEs) and CXEs was generated using RAxML software with 100 bootstrap replicates. All deduced amino acid sequences were obtained from GenBank: Drosophila melanogaster-neurotactin (NP_730196.1), Heliothis virescens-JHE (AAC38822.1), Helicoverpa armigera-JHE (AEB77712.1), Bombyx mori-JHE (AAR37335.1), Choristoneura fumiferana-JHE (AAD34172.1), Tenebrio molitor-JHE (AAL41023.1), Tribolium castaneum-JHE (BAJ10679.1), Psacothea hilaris-JHE (BAE94685.1), Gryllus assimilis-JHE (ABQ23214.1), Aedes aegypti-JHE (AAEL005200-PA), D. melanogaster-JHE (NP_001286476.1), D. melanogaster-JHEdup (NP_611085.2), Manduca sexta-JHE (AAG42021.2), Eriocheir sinensis-CXE1 (A0A1L5JHT6), E. sinensis-CXE2 (A0A1L5JHU7), Portunus trituberculatus-CXE1 (A0A1I9KY23), P. trituberculatus-CXE2 (A0A1I9KJ57), Pandalopsis japonica-CXE1 (F5A5Q6), P. japonica-CXE2 (F5A5Q7), Macrobrachium rosenbergii-CXE (MG910496.1). The neurotactin of D. melanogaster was used as an outgroup.

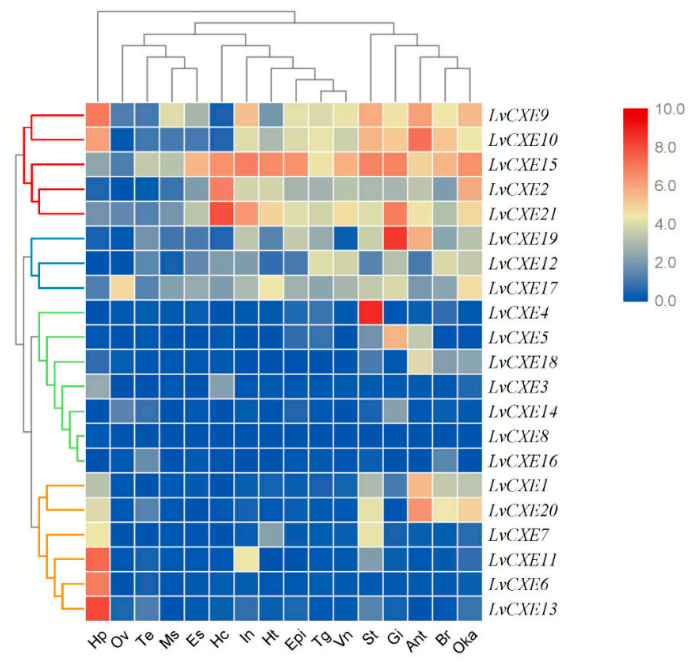

Figure 5. The expression profiles of LvCXEs in 16 adult tissues. Log2-transformed expression values were used to create the heat map. Red or blue colors represent the higher or lower relative abundance, respectively. Red, blue, green and yellow clades on the left representemodule 1-4, respectively, which were clustered by hierarchical clustering. LvCXEs shared similar expression profiles in each module. The bottom lists adult tissues of L. vannamei. (Hc: hemocyte, Ant: antennary gland, Ms: abdominal muscle, In: intestine, Ov: ovary, St: stomach, Oka: lymphoid organ, Gi: gill, Hp: hepatopancreas, Te: testis, Es: eyestalk, Br: brain, Tg: thoracic ganglion, Vn: ventral nerve, Epi: epidermis, Ht: heart). 


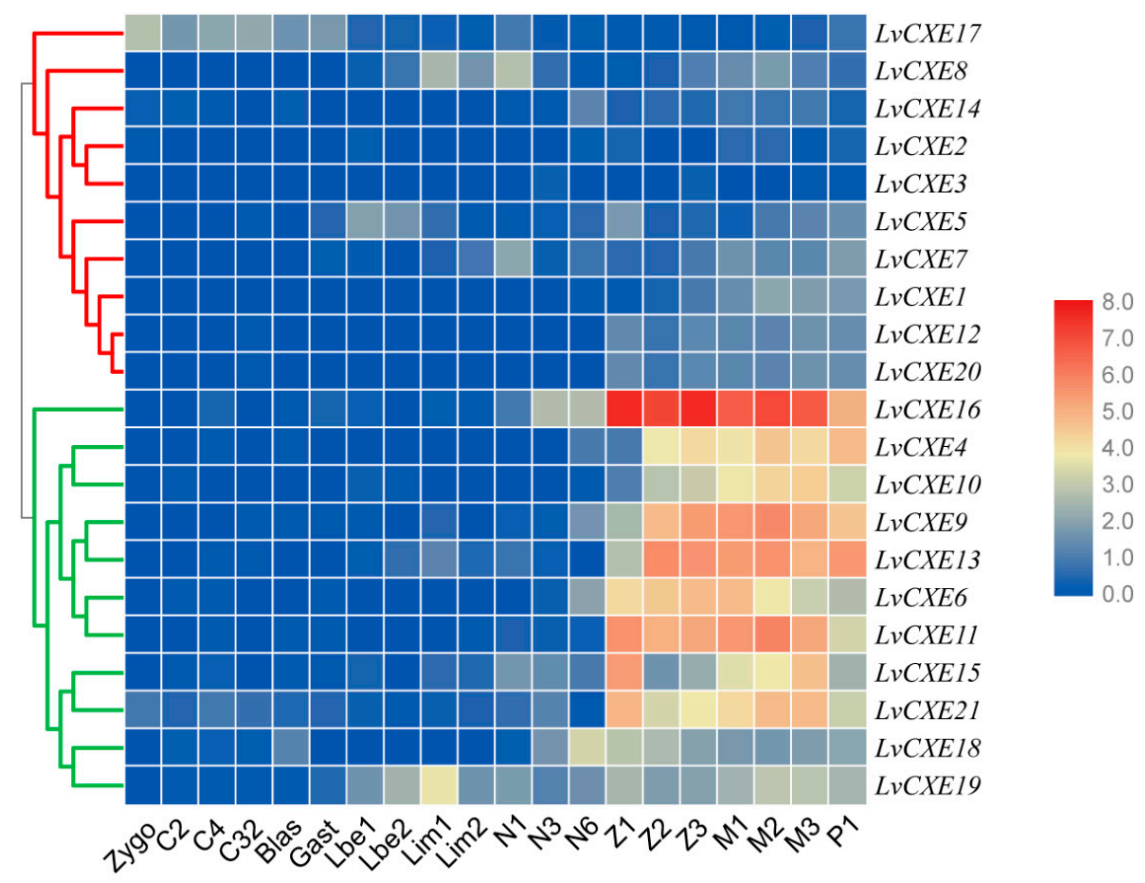

Figure 6. The expression profiles of LvCXEs during development of L. vannamei. Each rectangle with a white line represents the expression level of corresponding genes (right) in the corresponding stages (bottom). Blue and red colors represent low and high expressions, respectively. Red and green clades on the left represente module 1 and module 2, respectively, which were clustered by hierarchical clustering. LvCXEs shared similar expression profiles during the early development in each module. The bottom lists different larval stages of L. vannamei, from left to right—zygote, 2 cells (C2), 4 cells (C4), 32 cells (C32), blastula (blas), gastrula (gast), limb bud embryo I (Lbe1), limb bud embryo II (Lbe2), larva in membrane I (Lim1), larva in membrane II (Lim2), nauplius I (N1), nauplius II (N2), nauplius III (N3), nauplius VI (N6), zoea I (Z1), zoea II (Z2), zoea III (Z3), mysis I (M1), mysis II (M2), mysis III (M3) and post-larva $1(\mathrm{P} 1)$.

\subsection{Expression Patterns of LvCXEs during Molting}

All LvCXEs were detectable with dynamic expression patterns during the molting cycle. Module 1, including 16 LvCXEs, LvCXE1, LvCXE2, LvCXE4, LvCXE5, LvCXE6, LvCXE7, LvCXE9, LvCXE10, LvCXE11, LvCXE13, LvCXE16, LvCXE17, LvCXE18, LvCXE19, LvCXE20 and LvCXE21, showed similar expression patterns that the expression levels were high at $C$ and D0 stage, decreased in D3 stage, and increased rapidly in D4 stage during the molting period (Figure 7, Supplementary Figure S1). Furthermore, 11 of 14 GESAG-containing LvCXEs exhibited molt-dependent expressions, indicating that these expanded LvCXEs might be crucial for the molt cycle of $L$. vannamei. However, the results of hierarchical clustering analysis showed that the expression patterns of module 2, including LvCXE3, LvCXE8, LvCXE12, LvCXE14 and LvCXE15, were distinct from module 1. They exhibited low expressions in the C-D3 stage and rapid high expressions in D4 stage.

\subsection{OTFP Inhibition}

To further verify the relationship between LvCXEs and the molting process of L. vannamei, we conducted OTFP inhibition experiments. The real-time PCR results revealed that the expression levels of $13 \mathrm{LvCXEs}$ were significantly down-regulated in hepatopancreas after OTFP injection (Figure 8A). The average inhibition efficiency was about $55 \%$, and the highest was $88 \%$. The expression levels of $6 \mathrm{LvCXEs}$ showed no significant difference or were not detected. The molting times of each group were recorded and the cumulative molting rate was calculated every $24 \mathrm{~h}$ after injection. The results showed that a total of 14 shrimps of control group, accounting for $24.1 \%$, molted in 12 days. 
However, none of them molted successfully in the OTFP inhibition group. Moreover, eight shrimps died due to their exoskeletons being separated incompletely from the body, which resulted in unsuccessful molting process (Figure $8 \mathrm{~B}$ ). Additionally, many key ecdysone response genes were also affected by OTFP inhibition. The expressions of E75 (ecdysone-induced protein 75), Br-C (Broad-Complex), $\mathrm{Hr} 3$ (hormone receptor 3) and Ftz-f1 (Fushi tarazu factor-1) gene were all significantly down-regulated after OTFP inhibition (Figure 8C). Compared with the control group, the relative expressions of E75, Br-C, $\mathrm{Hr} 3$ and Ftz-f1 gene in OTFP inhibition group reduced by about $91 \%, 97 \%, 93 \%$ and $79 \%$, respectively.

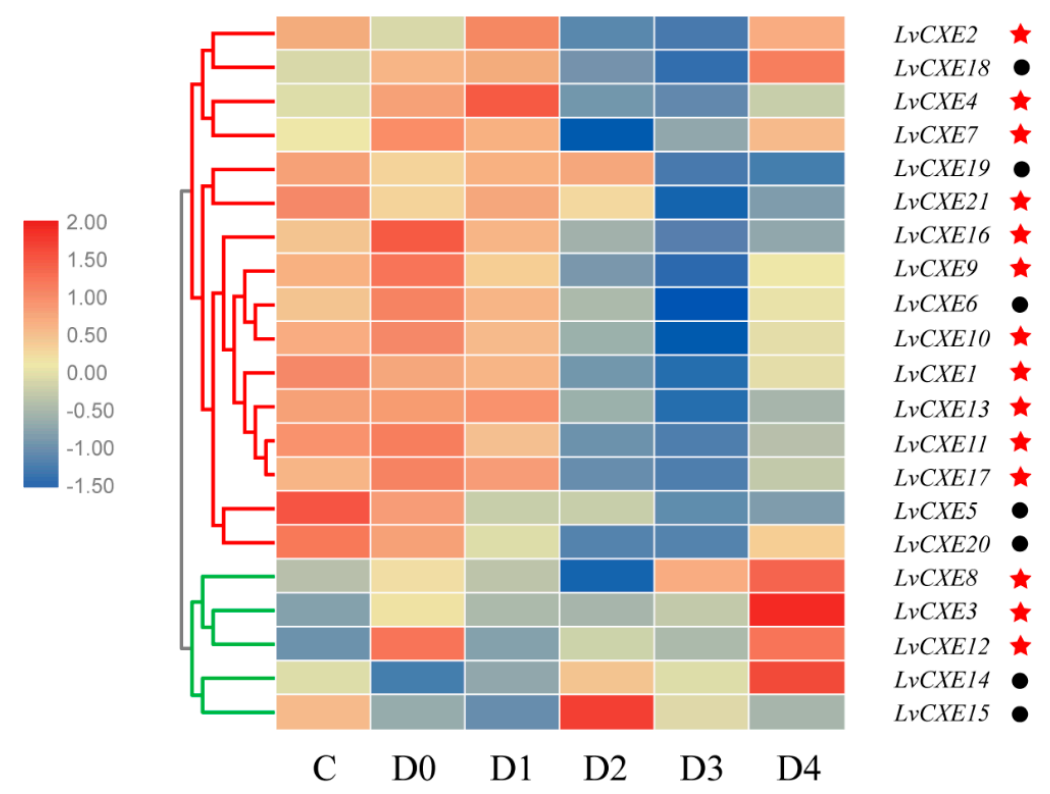

Figure 7. The expression profiles of LvCXEs during molt cycles of L. vannamei. Red and green clades represente module 1 and module 2, respectively, which were clustered by hierarchical clustering. LvCXEs shared similar expression profiles during the molt cycle in each module. The bottom lists different molting stages of L. vannamei, from left to right-inter-molt (C) and pre-molt (D0, D1, D2, D3, D4) stage. Red stars represent $L v C X E$ containing a GESAG motif and black circles represent $L v C X E$ s without a GESAG motif.

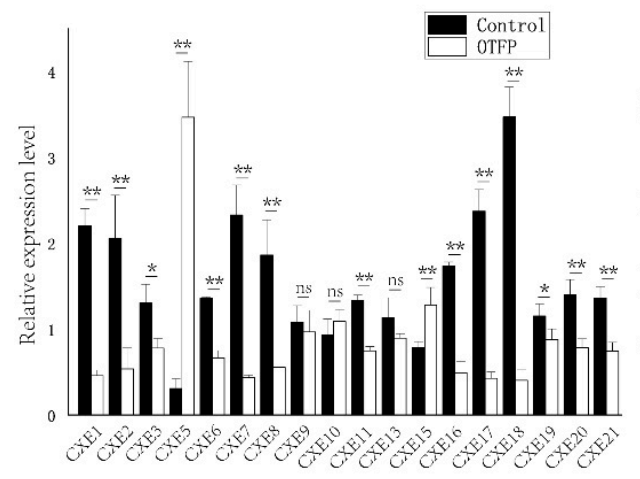

A

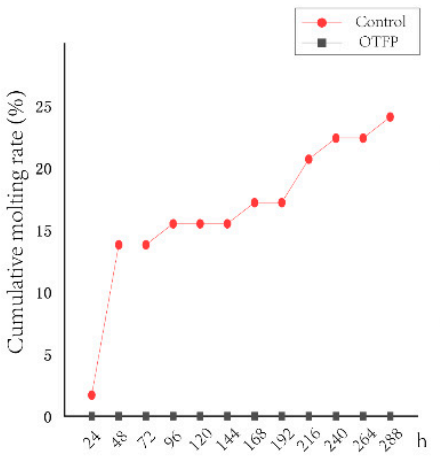

B

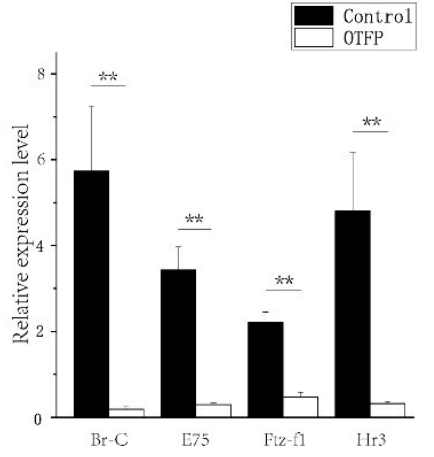

C

Figure 8. Effect of 3-octylthio-1,1,1-trifluoropropan-2-propanone (OTFP) inhibition on the cumulative molting rate and the expression of $L v C X E s, L v B r-C, L v E 75, L v F t z-f 1$ and $L v H r 3$. (A). The relative expression levels of LvCXEs after OTFP inhibition. (B). The cumulative molting rate in control group (red line) and OTFP group (grey line). (C). The relative expression levels of $L v B r-C, L v E 75, L v F t z-f 1$ and $\mathrm{LvHr} 3$ after OTFP inhibition. Significant differences in gene expression levels between two treatments were shown with a star $\left(^{*}\right)$ at $p<0.05$ or two stars $\left({ }^{* *}\right)$ at $p<0.01$. 'ns' represents no significant difference. 


\section{Discussion}

Previous studies have showed that a specific expansion of the CXE gene family was found in the L. vannamei genome through comparative genomic analysis [36]. Since we found seven, two and zero CXE genes in the genome of Procambarus virginalis, Parhyale hawaiensis and Daphnia duplex, L. vannamei has the largest number of CXE genes. The expansion of the gene family is supposed to strengthen the specific biological process or contribute to the adaptive evolution [36,37]. Hence, comprehensive analysis of the CXE gene family would not only study their function systematically, but also illustrate the relationship between its expansion and specific biological characteristics.

The features of sequences determine their biological functions. All LvCXEs identified in this study had conserved motifs and sites similar to those of insect JHEs or other crustacean CXEs. Furthermore, all CXE genes of crustaceans were clustered into a clade of the phylogenetics tree. These results indicated that all LvCXEs belong to the CXE family. Low bootstrap values of some branches may be caused by low sequence similarity between CXEs and JHEs, since there are no large conserved domains. In general, JHE containing a GQSAG motif can degrade JH in insects [38,39]. In this study, only LvCXE5 contained the GQSAG motif, but it was not clustered with insect JHEs in the phylogenetic tree and not expressed in the hepatopancreas or gonads. It indicated that LvCXE5 was not an ortholog of insect JHE. Meanwhile, previous studies found that CXE genes containing the GESAG motif were proposed to have esterase activity for MF in crustaceans [30,32]. Consistently, 14 LvCXEs contained GESAG in our study. Compared with JHE genes containing GQSAG and esterase activity for JH, the difference may be caused by the divergence between crustaceans and insects. Hence, we speculated that LvCXEs containing a GESAG motif may degrade MF in L. vannamei. However, since the study on CXE and MF in crustaceans is limited, it is arbitrary to conclude that all LvCXEs are involved in the degradation of MF just according to their sequence characteristics.

To explore the preliminary function of LvCXEs, we first analyzed their expressions profiles in various tissues. MF esterase activity has been detected in the hepatopancreas and gonads of many crustaceans $[40,41]$, which were considered to be the main sites for MF inactivation. In the present study, seven LvCXEs exhibited high expression in the hepatopancreas, but only LvCXE17 was expressed in the ovary. This is probably because the shrimp samples of different tissue transcriptomes are not in the stage of gonadal development. Interestingly, MF esterase activity has not been detected in the hemolymph of crustaceans so far [30], but we firstly found that three LvCXEs showed a high expression in hemocyte. Additionally, we firstly found that about half of $L v C X E$ s were expressed in the antennal gland, which provided clues for the significant role of MF and the antennal gland in osmoregulation [12,42]. Meanwhile, our study also provided clues for better understanding the diverse functions of CXE genes and MF in crustaceans.

Similar to the functions of JH in insects, MF inhibits the metamorphosis of crustaceans $[16,17]$. Hence, the expression levels of $L v C X E$ s were analyzed to further investigate their preliminary function during the developmental stage. Consistent with the increase of JHE activity in the late developmental

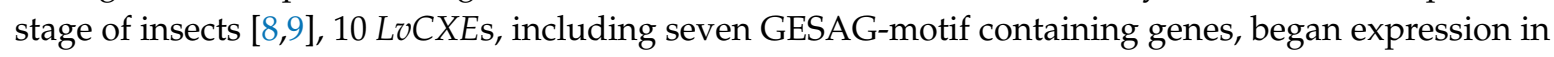
the nauplius, and were highly expressed in the zoeae and mysis stage gradually in L. vannamei. These may decrease MF titer and enable shrimp larvae metamorphosis to occur successfully during the early developmental stage. Furthermore, the decreased expression levels of LvCXEs at the post-larvae stage may restore MF titer. The above results reveal that $L v C X E$ s possess similar function to the JHE gene of insects during early development. However, L. vannamei has evolved a pattern that multiple LvCXEs control and compensate functionally to maintain normal metamorphosis simultaneously.

The study of the function of $L v C X E$ s during the molting cycle is of great significance since MF plays an important role in the molting process. In our study, most $L v C X E s$ presented molt-related expressions, suggesting the potential relationship between $L v C X E$ s and the molting process of $L$. vannamei. Under normal conditions, the expression levels of LvCXEs in module 1 increased significantly at D4 stage, which might result in a rapid decrease of MF titer and lead to the decrease of ecdysone in hemolymph. Similarly, the levels of MF, which fluctuate consistently with ecdysone [20-22], rise gradually at the early 
pre-molt stage but rapidly decline at the late pre-molt stage in the hemolymph of $M$. rosenbergii [43] and P. clarkia [15]. However, the injection of OTFP in D3 stage inhibited the transcription of LvCXEs and then might lead to the increase of MF titer rapidly, which elevated ecdysone titer in hemolymph abnormally. Furthermore, the synthesis of ecdysone in Y organ was then inhibited owing to the negative feedback, which was supported by the result that the expression levels of transcription factors $L v E 75, L v B r-C$, $L v H r 3$ and LvFtz-f1 were significantly down-regulated. Hence, the above results confirm that the high expression of LvCXEs in D4 stage is necessary for the molting cycle, and the CXE gene family plays an important role in the regulation of frequent molting in L. vannamei. However, MF exposure or MF injection accelerated the molting process of crustaceans according to previous studies $[15,18]$. We guess that they did not perform the MF treatment near molting, because they did not check the molting stage of the samples before the experiment. Hence, we speculate that MF can accelerate the molting process at the inter-molt or early pre-molt stage, but hinder the molting process near molting. Furthermore, it is also of concern that the expression patterns of module 2, including LvCXE3, LvCXE8, LvCXE12 LvCXE14 and LvCXE15, were different from module 1. Besides, the expression levels of LvCXE3, LvCXE8, LvCXE12 and LvCXE14 were very low not only in the hepatopancreas, but also at the early development stages. The above results showed that the function of these five LvCXEs should be different or divergent after the gene family expansion. However, there is no significant difference in the amino acid composition of their domains and key sites. It is speculated that the differences in their expression patterns are caused by different promoter regions. Of course, it needs more evidence to support this speculation.

\section{Materials and Methods}

\subsection{Genome and Transcriptome Data Resources}

The genomic data of L. vannamei were obtained from our laboratory (NCBI GenBank, PRJNA438564) [36]. Our previous research conducted RNA-seq on various libraries. Five larval stages, including embryo, nauplius, zoea, mysis and post-larvae [44]; eight molting stages, including inter-molt (C), pre-molt (D0, D1, D2, D3, D4) and post-molt (P1 and P2) stages [45]; and 16 adult tissues, including antennal gland, brain, hemocyte, epidermis, eyestalk, gill, hepatopancreas, heart, intestine, abdominal muscle, lymphoid organ, ovary, stomach, testis, thoracic ganglion and abdominal ganglion [46].

\subsection{The Characterization of CXE Gene Family in L. vannamei}

The CXE protein sequences of the morotoge shrimp Pandalopsis japonica (F5A5Q6), Chinese mitten crab Eriocheir sinensis (A0A1L5JHT6) and Drosophila melanogaster-JHE (NP_001286476.1) were used as query to search against all unigene datasets by tblastn (E-value $\leq 10^{-6}$ ) and the whole genome predicted proteins by blastp $\left(\mathrm{E}\right.$-value $\left.\leq 10^{-6}\right)$. Then, redundant sequences were removed using the CAP3 program [47]. The candidate sequences were submitted to ORF Finder (https://www.ncbi.nlm. nih.gov/orffinder/) and the ExPASy translate tool (http://web.expasy.org/translate/) to obtain deduced amino acid sequences. The integrity of the carboxylesterase domain was predicted using SMART (http: //smart.embl-heidelberg.de/) and InterPro database (http://www.ebi.ac.uk/interpro/) (E-value $\leq 10^{-50}$ ). The presence of a signal peptide was analyzed by SignalP 4.1 server (www.cbs.dtu.dk/services/SignalP/). A multiple alignment was performed using ClustalW [48] and visualized by jalview [49]. LvCXEs were identified if they contained the following three structures-(1) a putative signal peptide in N-terminus (apart from incomplete sequences), (2) conserved catalytic triad (Ser-Glu-His) residues, and (3) a CXE-specific GxSxG motif. The theoretical isoelectric point (pI) and molecular weight (Mw) were calculated using online tool (http://web.expasy.org/compute_pi/). The conserved motifs were analyzed using the MEME program (http://meme-suite.org/) with default parameters [50] and visualized by TBtools [51]. To determine the locations of LvCXEs in L. vannamei genome, all LvCXE sequences were mapped to the whole genome scaffolds by blastn program (E-value $\leq 10^{-30}$ ). The exon/intron positions 
were then determined when the identity of aligned region $>97 \%$. The exon/intron boundaries were manually checked and the structures were finally visualized with the Gene Structure Display Server web tool (http:/gsds.cbi.pku.edu.cn/).

\subsection{Phylogenetic Analysis}

The full-length amino acid sequences of CXE from crustaceans and JHE from insects were collected from GenBank databases. All collected sequences were aligned using MUSCLE program (v3.8.31) [52] with default parameters. Then the best-fit model for constructing the phylogenetic tree was selected by ProtTest 3.4.2 (parameter: -all-distributions -F -AIC -BIC -tc 0.5) [53]. A maximum likelihood (ML) phylogenetic tree of LvCXEs was generated by RAxML 8.0.26 [54] with a WAG+I+G+F model. Meanwhile, the ML phylogenetic tree of arthropod JHEs/CXEs was generated with a WAG+G+F model. All trees were tested with 100 bootstrap replicates and finally visualized using iTOL [55].

\subsection{Expression Profiles}

In previous studies, the RPKM (reads per kilobases per million reads) values of all transcripts of 20 early development stages, 8 molting stages and 16 adult tissues were calculated [44-46]. The RPKM values of all $L v C X E$ s were extracted and then normalized with $\log 2$ conversion. Heat maps were then created and clustered with hierarchical clustering using TBtools software [51].

\subsection{OTFP Inhibition}

To investigate the relationships between LvCXEs and the molting process of L. vannamei, a total of 120 shrimps (about $5 \mathrm{~cm}$ for body length) in D0-D4 stage were selected and maintained in filtered seawater at a temperature of $25 \pm 1{ }^{\circ} \mathrm{C}$ with continuous aeration. Before the experiment, all shrimps were kept in the aquarium for 3 days to acclimate them to the laboratory conditions. They were then randomly selected into a control group and experimental group. For each group, three replicates were conducted. The control group was injected with $10 \mu \mathrm{L}$ mixture of ethanol and PBS (volume ratio 3:7) and the experimental group was injected with $10 \mu \mathrm{L} 4.19 \times 10^{-3} \mathrm{~mol} / \mathrm{L}$ OTFP solution, which was diluted by the mixture of ethanol and PBS (volume ratio 3:7). The molting times of each group were recorded and the cumulative molting rate was calculated every $24 \mathrm{~h}$ after injection. To further quantify the expression levels of some molt-related genes, 20 shrimps in D3-D4 stage were randomly selected into a control group and experimental group. The control group was injected with $10 \mu \mathrm{L}$ mixture of ethanol and PBS (volume ratio 3:7) and the experimental group was injected with $10 \mu \mathrm{L}$ $4.19 \times 10^{-3} \mathrm{~mol} / \mathrm{L}$ OTFP solution, which was diluted by the mixture of ethanol and PBS (volume ratio 3:7). At $48 \mathrm{~h}$ postinjection, the hepatopancreas of four shrimps from each group was collected separately for RNA extraction.

\subsection{RNA-Isolation and $q R T-P C R$}

Total RNA was extracted using RNAiso Plus reagent (TaKaRa, Kyoto, Japan) according to the manufacturer's instructions. Agarose electrophoresis and NanoDrop 2000 (Thermo Fisher Scientific, Waltham, MA, USA) were used to detect the quality and the concentration of RNA, respectively. First-strand cDNA was synthesized using $1 \mu \mathrm{g}$ of total RNA with a PrimeScript RT reagent kit (TaKaRa). According to the instruction, the mixed primer included Oligo $\mathrm{d}(\mathrm{T})$ primer and random hexamer primer was used and the genomic DNA had been removed by DNase treatment for cDNA synthesis. cDNA was synthesized according to the following procedure $-37^{\circ} \mathrm{C}$ for $1 \mathrm{~h}$, and $85^{\circ} \mathrm{C}$ for $5 \mathrm{~s}$. The cDNA samples were stored at $-80^{\circ} \mathrm{C}$ for further use.

SYBR Green-based quantitative real-time PCR (qRT-PCR) was performed to detect the expression levels of $L v C X E$ s and four ecdysone response genes in the hepatopancreas using THUNDERBIRD ${ }^{\mathrm{TM}}$ SYBR $^{\circledR}$ (TOYOBO, Osaka, Japan). Four technical replicates were conducted for each gene to eliminate the systematic errors. The $18 \mathrm{~S}$ rRNA was used as an internal standard. Primer sequences were listed in Supplementary Table S1. qPCR was performed with an Eppendorf Mastercycler ep realplex 
(Eppendorf, Hamburg, Germany) using the following program-denaturation at $95^{\circ} \mathrm{C}$ for $15 \mathrm{~min}$; 40 cycles of $95^{\circ} \mathrm{C}$ for $15 \mathrm{~s}$, annealing temperature for $15 \mathrm{~s}$, and $72{ }^{\circ} \mathrm{C}$ for $20 \mathrm{~s}$. The PCR melting-curve was used to check the specificity of the PCR product. In addition, relative expression levels were calculated with the formula $2^{-\Delta \Delta C}$ T using the comparative $C_{\mathrm{T}}$ method [56]. An unpaired two-tailed t test was used for statistical analysis by $\mathrm{R}$ (version 3.3.3). $p$ value $<0.05$ was considered statistically significant.

\section{Conclusions}

In summary, a total of 21 complete $L v C X E$ s were identified in the L. vannamei genome. Their genome localizations, gene structures and conserved domains were comprehensively analyzed. The CXE genes with a GESAG motif expanded specifically in the L. vannamei genome, which increased the diversity of gene structures and functions. At the end of the early developmental stage, the high expression levels of LvCXEs may enable shrimp larvae to develop successfully. The high expression levels of LvCXEs in D4 stage are essential for the molting cycle. In contrast, the abnormal function of LvCXEs will lead to the failure of the molting process. In conclusion, the expansion of the CXE gene family is related to precise regulation for early development and frequent molting in L. vannamei.

Supplementary Materials: Supplementary Materials can be found at http://www.mdpi.com/1422-0067/21/15/ 5444/s1. Figure S1: The normalized expressions of LvCXEs in module 1 of Figure 7. Each line represents the expression level of a LvCXE in module 1. Table S1: Primers for real-time qRT-PCR.

Author Contributions: F.L., J.X. and X.Z. (Xiaojun Zhang) conceived and designed the study. X.Z. (Xiaoxi Zhang) collected the data, conducted the bioinformatics analyses, conducted all experiments and wrote the manuscript. J.Y., X.Z. (Xiaojun Zhang) and F.L. revised the manuscript. All authors have read and agreed to the published version of the manuscript.

Funding: This research was funded by the National Key R\&D Program of China (2018YFD0900103), the National Natural Sciences Foundation of China (31672632, 31972782 and 41876167) and China Agriculture Research System-48 (CARS-48).

Acknowledgments: OTFP used in this study was provided by Xiaojun Gu from Fujian Agricultural and Forestry University.

Conflicts of Interest: The authors declare no conflict of interest.

\section{References}

1. Wu, X.M.; Xu, B.Y.; Si, F.L.; Li, J.; Yan, Z.T.; Yan, Z.W.; Xiu, H.; Chen, B. Identification of carboxylesterase genes associated with pyrethroid resistance in the malaria vector Anopheles sinensis (Diptera: Culicidae). Pest Manag. Sci. 2017, 74, 159-169. [CrossRef] [PubMed]

2. Yu, Q.Y.; Cheng, L.; Li, W.L.; Xiang, Z.H.; Zhang, Z. Annotation and expression of carboxylesterases in the silkworm, Bombyx mori. BMC Genom. 2009, 10, 553. [CrossRef]

3. Hilary, R.; Charles, C.; Federica, O.; Christelle, A.; Janet, H.; Sharakhova, M.V.; Unger, M.F.; Collins, F.H.; René, F. Evolution of supergene families associated with insecticide resistance. Science 2002, 298, 179-181.

4. Gilbert, L.I.; Granger, N.A.; Roe, R.M. The juvenile hormones: Historical facts and speculations on future research directions. Insect Biochem. Mol. Biol. 2000, 30, 617-644. [CrossRef]

5. Tobe, S.S.; Bendena, W.G. The Regulation of Juvenile Hormone Production in Arthropods: Functional and Evolutionary Perspectives. Ann. N. Y. Acad. Sci. 2006, 897, 300-310. [CrossRef]

6. Venkatesh, K.; Roe, R.M.; Apperson, C.S.; Sonenshine, D.E.; Schriefer, M.E.; Boland, L.M. Metabolism of juvenile hormone during adult development of Dermacentor variabilis (Acari: Ixodidae). J. Med. Entomol. 1990, 27, 36-42. [CrossRef] [PubMed]

7. Wyatt, G.R.; Davey, K.G. Cellular and molecular actions of juvenile hormone. II. Roles of juvenile hormone in adult insects. Adv. Insect Physiol. 1996, 26, 1-155.

8. Hirai, M.; Kamimura, M.; Kikuchi, K.; Yasukochi, Y.; Kiuchi, M.; Shinoda, T.; Shiotsuki, T. cDNA cloning and characterization of Bombyx mori juvenile hormone esterase: An inducible gene by the imidazole insect growth regulator KK-42. Insect Biochem. Mol. Biol. 2002, 32, 627-635. [CrossRef] 
9. Yang, H.J.; Zhou, F.; Awquib, S.; Malik, F.A.; Roy, B.; Li, X.H.; Hu, J.B.; Sun, C.G.; Niu, Y.S.; Miao, Y.G. Expression pattern of enzymes related to juvenile hormone metabolism in the silkworm, Bombyx mori L. Mol. Biol. Rep. 2011, 38, 4337-4342.

10. Kamita, S.G.; Hinton, A.C.; Wheelock, C.E.; Wogulis, M.D.; Wilson, D.K.; Wolf, N.M.; Stok, J.E.; Hock, B.; Hammock, B.D. Juvenile hormone (JH) esterase: Why are you so JH specific? Insect Biochem. Mol. Biol. 2003, 33, 1261-1273. [CrossRef]

11. Abdel-Aal, Y.A.; Hammock, B.D. Transition state analogs as ligands for affinity purification of juvenile hormone esterase. Science 1986, 233, 1073-1076. [CrossRef] [PubMed]

12. Nagaraju, G.P.C. Is methyl farnesoate a crustacean hormone? Aquaculture 2007, 272, 39-54. [CrossRef]

13. Xie, X.; Tao, T.; Liu, M.; Zhou, Y.; Liu, Z.; Zhu, D. The potential role of juvenile hormone acid methyltransferase in methyl farnesoate (MF) biosynthesis in the swimming crab, Portunus trituberculatus. Anim. Reprod. Sci. 2016, 168, 40-49. [CrossRef]

14. Li, S.; Friesen, J.A.; Holford, K.C.; Borst, D.W. Methyl farnesoate synthesis in the lobster mandibular organ: The roles of HMG-CoA reductase and farnesoic acid O-methyltransferase. Comp. Biochem. Physiol. Part A Mol. Integr. Physiol. 2010, 155, 49-55. [CrossRef]

15. Laufer, H.; Demir, N.; Pan, X.; Stuart, J.D.; Ahl, J.S. Methyl farnesoate controls adult male morphogenesis in the crayfish, Procambarus clarkii. J. Insect Physiol. 2005, 51, 379-384. [CrossRef]

16. Borst, D.W.; Laufer, H.; Landau, M.; Chang, E.S.; Hertz, W.A.; Baker, F.C.; Schooley, D.A. Methyl farnesoate and its role in crustacean reproduction and development. Insect Biochem. 1987, 17, 1123-1127. [CrossRef]

17. Abdu, U.; Takac, P.; Laufer, H.; Sagi, A. Effect of Methyl Farnesoate on Late Larval Development and Metamorphosis in the Prawn Macrobrachium rosenbergii (Decapoda, Palaemonidae): A Juvenoid-Like Effect? Biol. Bull. 1998, 195, 112-119. [CrossRef]

18. Abdu, U.; Barki, A.; Karplus, I.; Barel, S.; Takac, P.; Yehezkel, G.; Laufer, H.; Sagi, A. Physiological effects of methyl farnesoate and pyriproxyfen on wintering female crayfish Cherax quadricarinatus. Aquaculture 2001, 202, 163-175. [CrossRef]

19. Ahl, J.S.B.; Brown, J.J. Salt-dependent effects of juvenile hormone and related compounds in larvae of the brine shrimp, Artemia. Comp. Biochem. Physiol. Part A Mol. Integr. Physiol. 1990, 95, 491-496. [CrossRef]

20. Wang, C.J.; Zhu, D.F.; Qi, Y.Z.; Hu, Z.H.; Shen, J. Molt-inhibiting hormone levels and ecdysteroid titer during a molt cycle of Portunus trituberculatus. Acta Hydrobiol. Sin. 2013, 37, 22-28.

21. Xu, Y.; Jian, L.; Yanting, C.; Li, M. Changes in Immunity-Related Enzymes, Chitinase Activity, and Molting Ecdysteroid Concentration of Exopalaemon carinicauda during Different Molting Stages. Prog. Fish. Sci. 2018, 39, 120-125.

22. Lee, K.J. Molt-inhibiting hormone mRNA levels and ecdysteroid titer during a molt cycle of the blue crab, Callinectes sapidus. Biochem. Biophys. Res. Commun. 1998, 249, 624-627. [CrossRef]

23. Chang, E.S.; Mykles, D.L. Regulation of crustacean molting: A review and our perspectives. Gen. Comp. Endocrinol. 2011, 172, 323-330. [CrossRef]

24. Qu, Z.; Kenny, N.J.; Lam, H.M.; Chan, T.F.; Chu, K.H.; Bendena, W.G.; Tobe, S.S.; Hui, J.H.L. How Did Arthropod Sesquiterpenoids and Ecdysteroids Arise? Comparison of Hormonal Pathway Genes in Noninsect Arthropod Genomes. Genome Biol. Evol. 2015, 7, 1951-1959.

25. Song, Y.; Villeneuve, D.L.; Toyota, K.; Iguchi, T.; Tollefsen, K.E. Ecdysone Receptor Agonism Leading to Lethal Molting Disruption in Arthropods: Review and Adverse Outcome Pathway Development. Environ. Sci. Technol. 2017, 51, 4142-4157. [CrossRef]

26. Cheng, J.-H.; Chang, E.S. Ecdysteroid treatment delays ecdysis in the lobster, Homarus americanus. Biol. Bull. 1991, 181, 169-174. [CrossRef]

27. Schafellner, C.; Eizaguirre, M.; López, C.; Sehnal, F. Juvenile hormone esterase activity in the pupating and diapausing larvae of Sesamia nonagrioides. J. Insect Physiol. 2008, 54, 916-921. [CrossRef]

28. Elayidam, U.G.; Muraleedharan, D. Juvenile hormone activity in Dysdercus cingulatus Fabr by juvenile hormone esterase inhibitor, OTFP. Indian J. Exp. Biol. 2007, 45, 901-906.

29. Takac, P.; Ahl, J.S.B.; Laufer, H. Seasonal differences in methyl farnesoate esterase activity in tissues of the spider crab Libinia emarginata. Int. J. Invertebr. Reprod. Dev. 1997, 31, 211-216. [CrossRef]

30. Tao, T.; Xie, X.; Liu, M.; Jiang, Q.; Zhu, D. Cloning of two carboxylesterase cDNAs from the swimming crab Portunus trituberculatus: Molecular evidences for their putative roles in methyl farnesotae degradation. Comp. Biochem. Physiol. Part B Biochem. Mol. Biol. 2017, 203, 100-107. [CrossRef] 
31. Zhu, X.J.; Xiong, Y.; He, W.; Jin, Y.; Qian, Y.Q.; Liu, J.; Dai, Z.M. Molecular cloning and expression analysis of a prawn (Macrobrachium rosenbergii) juvenile hormone esterase-like carboxylesterase following immune challenge. Fish Shellfish Immunol. 2018, 80, 10-14. [CrossRef] [PubMed]

32. Xu, Y.; Zhao, M.; Deng, Y.; Yang, Y.; Li, X.; Lu, Q.; Ge, J.; Pan, J.; Xu, Z. Molecular cloning, characterization and expression analysis of two juvenile hormone esterase-like carboxylesterase cDNAs in Chinese mitten crab, Eriocheir sinensis. Comp. Biochem. Physiol. Part B Biochem. Mol. Biol. 2017, 205, 46-53. [CrossRef] [PubMed]

33. Lee, S.O.; Jeon, J.M.; Oh, C.W.; Kim, Y.M.; Kang, C.K.; Lee, D.S.; Mykles, D.L.; Kim, H.W. Two juvenile hormone esterase-like carboxylesterase cDNAs from a Pandalus shrimp (Pandalopsis japonica): Cloning, tissue expression, and effects of eyestalk ablation. Comp. Biochem. Physiol. Part B Biochem. Mol. Biol. 2011, 159, 148-156. [CrossRef]

34. Godin, D.M.; Carr, W.H.; Hagino, G.; Segura, F.; Sweeney, J.N.; Blankenship, L. Evaluation of a fluorescent elastomer internal tag in juvenile and adult shrimp Penaeus vannamei. Aquaculture 1996, 139, $243-248$. [CrossRef]

35. Gao, Y.; Wei, J.; Yuan, J.; Zhang, X.; Li, F.; Xiang, J. Transcriptome analysis on the exoskeleton formation in early developmetal stages and reconstruction scenario in growth-moulting in Litopenaeus vannamei. Sci. Rep. 2017, 7, 1098. [CrossRef]

36. Zhang, X.; Yuan, J.; Sun, Y.; Li, S.; Gao, Y.; Yu, Y.; Liu, C.; Wang, Q.; Lv, X.; Zhang, X.; et al. Penaeid shrimp genome provides insights into benthic adaptation and frequent molting. Nat. Commun. 2019, 10, 356. [CrossRef]

37. Zhang, G.; Fang, X.; Guo, X.; Li, L.; Luo, R.; Xu, F.; Yang, P.; Zhang, L.; Wang, X.; Qi, H. The oyster genome reveals stress adaptation and complexity of shell formation. Nature 2012, 490, 49-54. [CrossRef] [PubMed]

38. Duan, D.; Zheng, R.; Lin, S.; Chen, Y.; Tian, H.; Zhao, J.; Tian, S.; Wei, H.; Gu, X. Modulation of Juvenile Hormone Esterase Gene Expression Against Development of Plutella xylostella (Lepidoptera: Plutellidae). J. Econ. Entomol. 2016, 109, 865-872. [CrossRef]

39. Wogulis, M.; Wheelock, C.E.; Kamita, S.G.; Hinton, A.C.; Whetstone, P.A.; Hammock, B.D.; Wilson, D.K. Structural Studies of a Potent Insect Maturation Inhibitor Bound to the Juvenile Hormone Esterase of Manduca sexta. Biochemistry 2006, 45, 4045-4057. [CrossRef]

40. Homola, E.; Chang, E.S. Methyl Farnesoate: Crustacean Juvenile Hormone in Search of Functions. Comp. Biochem. Physiol. Part B Biochem. Mol. Biol. 1997, 117, 347-356. [CrossRef]

41. Homola, E.; Chang, E.S. Distribution and regulation of esterases that hydrolyze methyl farnesoate in Homarus americanus and other crustaceans. Gen. Comp. Endocrinol. 1997, 106, 62-72. [CrossRef] [PubMed]

42. Tsai, J.-R.; Lin, H.-C. Functional anatomy and ion regulatory mechanisms of the antennal gland in a semi-terrestrial crab, Ocypode stimpsoni. Biol. Open 2014, 3, 409-417. [CrossRef] [PubMed]

43. Wilder, M.N.; Okada, S.; Fusetani, N.; Aida, K. Hemolymph Profiles of Juvenoid Substances in the Giant Freshwater Prawn Macrobrachium rosenbergii in Relation to Reproduction and Molting. Fish. Sci. 1995, 61, 175-176. [CrossRef]

44. Wei, J.; Zhang, X.; Yu, Y.; Huang, H.; Li, F.; Xiang, J. Comparative Transcriptomic Characterization of the Early Development in Pacific White Shrimp Litopenaeus vannamei. PLoS ONE 2014, 9, e106201. [CrossRef]

45. Gao, Y.; Zhang, X.; Wei, J.; Sun, X.; Yuan, J.; Li, F.; Xiang, J. Whole Transcriptome Analysis Provides Insights into Molecular Mechanisms for Molting in Litopenaeus vannamei. PLoS ONE 2015, 10, e0144350. [CrossRef]

46. Zhang, X.; Zhang, X.; Yuan, J.; Du, J.; Li, F.; Xiang, J. Actin genes and their expression in pacific white shrimp, Litopenaeus vannamei. Mol. Genet. Genom. 2018, 293, 479-493. [CrossRef]

47. Huang, X.; Madan, A. CAP3: A DNA sequence assembly program. Genome Res. 1999, 9, 868-877. [CrossRef]

48. Wilm, A.; Higgins, D.G.; Valentin, F.; Blackshields, G.; McWilliam, H.; Wallace, I.M.; Thompson, J.D.; Larkin, M.A.; Brown, N.P.; McGettigan, P.A.; et al. Clustal W and Clustal X version 2.0. Bioinformatics 2007, 23, 2947-2948.

49. Waterhouse, A.M.; Martin, D.M.A.; Barton, G.J.; Procter, J.B.; Clamp, M. Jalview Version 2-A multiple sequence alignment editor and analysis workbench. Bioinformatics 2009, 25, 1189-1191. [CrossRef]

50. Bailey, T.L.; Williams, N.; Misleh, C.; Li, W.W. MEME: Discovering and analyzing DNA and protein sequence motifs. Nucleic Acids Res. 2006, 34, 369-373. [CrossRef]

51. Chen, C.; Chen, H.; Zhang, Y.; Thomas, H.R.; Frank, M.H.; He, Y.; Xia, R. TBtools-An integrative toolkit developed for interactive analyses of big biological data. Mol. Plant 2020. [CrossRef] [PubMed] 
52. Edgar, R.C. MUSCLE: Multiple sequence alignment with high accuracy and high throughput. Nucleic Acids Res. 2004, 32, 1792-1797. [CrossRef] [PubMed]

53. Abascal, F.; Zardoya, R.; Posada, D. ProtTest: Selection of best-fit models of protein evolution. Bioinformatics 2005, 21, 2104-2105. [CrossRef]

54. Alexandros, S. RAxML version 8: A tool for phylogenetic analysis and post-analysis of large phylogenies. Bioinformatics 2014, 30, 1312-1313.

55. Letunic, I.; Bork, P. Interactive Tree Of Life (iTOL) v4: Recent updates and new developments. Nucleic Acids Res. 2019, 47, W256-W259. [CrossRef] [PubMed]

56. Livak, K.J.; Schmittgen, T.D. Analysis of relative gene expression data using real-time quantitative PCR and the $2^{-\Delta \Delta C}$ T Method. Methods 2001, 25, 402-408. [CrossRef]

(C) 2020 by the authors. Licensee MDPI, Basel, Switzerland. This article is an open access article distributed under the terms and conditions of the Creative Commons Attribution (CC BY) license (http://creativecommons.org/licenses/by/4.0/). 